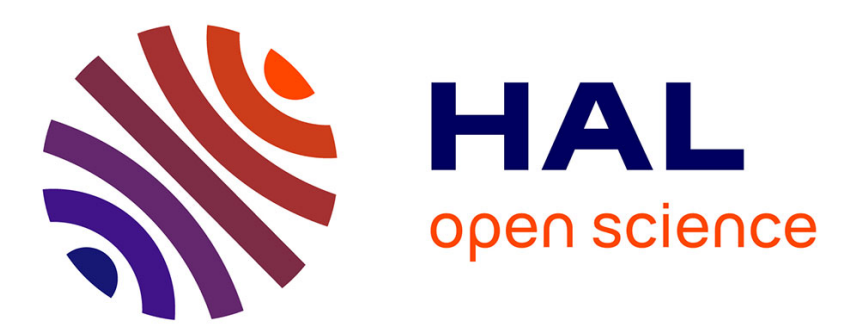

\title{
Efficient numerical algorithm to simulate a 3D coupled Maxwell-plasma problem
}

\author{
Adnane Hamiaz, Xavier Ferrì̀res, Olivier Pascal
}

\section{To cite this version:}

Adnane Hamiaz, Xavier Ferrìres, Olivier Pascal. Efficient numerical algorithm to simulate a 3D coupled Maxwell-plasma problem. Mathematics and Computers in Simulation, 2020, 174, pp.19-31. 10.1016/j.matcom.2020.02.018 . hal-02526812

\section{HAL Id: hal-02526812 \\ https://hal.science/hal-02526812}

Submitted on 24 Jul 2020

HAL is a multi-disciplinary open access archive for the deposit and dissemination of scientific research documents, whether they are published or not. The documents may come from teaching and research institutions in France or abroad, or from public or private research centers.
L'archive ouverte pluridisciplinaire HAL, est destinée au dépôt et à la diffusion de documents scientifiques de niveau recherche, publiés ou non, émanant des établissements d'enseignement et de recherche français ou étrangers, des laboratoires publics ou privés. 


\title{
Efficient Numerical Algorithm to Simulate a 3D Coupled Maxwell-Plasma Problem
}

\author{
Adnane Hamiaz* \\ Department of Mathematics, Faculty of Science, Taibah University, Al-Madinah \\ Al-Munawarah, Saudi Arabia. \\ Xavier Ferrieres \\ DEMR - Université Fédérale de Toulouse - ONERA, 2 avenue Edouard Belin, 31055 \\ Toulouse, France \\ Olivier Pascal \\ LAPLACE, Université Fédérale de Toulouse, 118, route de Narbonne, 31062 Toulouse, \\ France
}

\begin{abstract}
This paper proposes an improved algorithm, based upon an explicit finite difference scheme, in order to simulate the plasma breakdown induced by a monochromatic High Power Micro-Wave (HPM). The 3D coupled Maxwell-plasma equations are to be solved. We want to study with this model the geometry of the discharge and plasma formation at high pressure which may contribute to shield microwave sensors or circuits. Generally, the simulation of this kind of problem is very time-consuming, but by using the fact that the plasma evolution in time is slow relatively to the monochromatic source period, we can drastically reduce the simulation time. By considering this assumption, we describe in the paper a process which allows to obtain this important reduction. Finally, an example where we show the gain obtained in terms of computation time with our process is given to validate and illustrate the global work.
\end{abstract}

Keywords: Maxwell's equations, fluid plasma model, time-domain

\footnotetext{
* Corresponding author

Email addresses: ahamiaz@taibahu.edu.sa (Adnane Hamiaz), xavier.ferrieres@onera.fr (Xavier Ferrieres), olivier.pascal@laplace.univ-tlse.fr (Olivier Pascal)
}

Preprint submitted to Journal of Mathematics and Computers in Simulation. July 26, 2019 
simulations, Yee's scheme and finite-difference methods.

\section{Introduction}

In this paper, we are interested in the breakdown induced by an electromagnetic HPM (High Power Micro-Wave). An efficient numerical method is proposed, based upon a finite differences scheme, to reduce the computation 5 time in the simulation of a 3D coupled Maxwell-Plasma problem. In particular, we are interested in studying the microwave breakdowns on small structures and plasma formation at high pressure.

Such a kind of problem presents a big interest in ElectroMagnetic Compatibility (EMC) for the evaluation of the electromagnetic fields induced by an external source, on electronic components located inside a cavity which is not closed. By considering a HPM source, the important value of the electric field can create discharges. They are located on the small apertures or on the thin slots of the cavity. They are able to protect the inside components by reducing the value of the electromagnetic fields. The absorbing and reflecting properties of the plasma medium then enhance the shielding but it can also deteriorate dielectric materials in the long time as it has been observed in the context of partial discharges.

In the first part of the paper, we recall the equations of the studied physical problem proposed by J.P.Boeuf to solve the plasma formation at high pressure in a $2 \mathrm{D}$ context [1. In the second part, a $3 \mathrm{D}$ approximation to simulate this physical problem is detailed. This approximation, already developed in a $2 \mathrm{D}$ context [1] 2], is based on an explicit finite differences scheme and presents an important computational cost due to the different time and space characteristics of the electromagnetic problem and of the plasma problem. Indeed, to ensure an accurate solution for the plasma problem, it is mandatory to use a very small step size in space which implies for the Maxwell problem a very small time step to ensure stability. In the 3D case, this drawback involves an excessive computing time to treat efficiently the entire problem. To avoid this drawback, 
some authors have proposed an implicit numerical scheme to solve the Maxwell problem [3] 4, but this solution don't take into account the great diversity of physical model available in the Yee scheme for thin wires, slots, ..., needed in many of our applications. Therefore, we propose an optimized explicit numerical scheme where the new idea is to reduce the cost of the electromagnetic fields computation by avoiding to evaluate these fields at each step of the scheme. A quasi stationary state is assumed for the plasma medium to extract the fields in an appropriate number of periods. This allows to update the plasma density with a much longer time step without calculating electromagnetic fields all along. In the third part of this paper, we give the principle of this optimization and an example to validate this approach and evaluate the gain in terms of computation 40 time.

\section{Physical model}

The mathematical formulation of the physical model, taken into account for our study, is based upon a coupling between Maxwell's equations and plasma's equations.

In the literature, a lot of papers are dealing about the resolution of this system of equations. In particular, we can cite, the works of Yu [5] and Simpson [6] for studying the electromagnetic waves propagation inside a magnetized plasma. However, concerning our applications with a non magnetized medium, the most interesting model is the one of Boeuf [1] [7, where the mathematical 50 formulation is given by :

$$
\begin{aligned}
& \mu_{0} \frac{\partial \mathbf{H}}{\partial t}+\nabla \times \mathbf{E}=0 \\
& \epsilon_{0} \frac{\partial \mathbf{E}}{\partial t}-\nabla \times \mathbf{H}=-\mathbf{J} \\
& \mathbf{J} \simeq q u \mathbf{v}
\end{aligned}
$$

where $(\mathbf{E}, \mathbf{H})$ are the electromagnetic fields, $\mathbf{J}$ the current density, and $e, q=-e$, $u, \mathbf{v}$ are respectively the elementary charge, the electron charge, the density and the mean velocity. The electron velocity is obtained from a simplified 
momentum transfer equation:

$$
\frac{\partial \mathbf{v}}{\partial t}+\nu_{c} \mathbf{v}=\frac{q}{m_{e}} \mathbf{E}
$$

where $m_{e}$ is the electron mass, $\nu_{c}=5.3 \times 10^{9} p_{r}$ is the electron-neutral collision frequency in air and $p_{r}$ is the pressure in torr.

The plasma is assumed to be quasi-neutral $\left(n_{e}=n_{i}=u\right)$ and the time evolution of the plasma density $u$ is described by the diffusion equation :

$$
\frac{\partial u}{\partial t}-\nabla \cdot\left(D_{e f f} \nabla u\right)=\nu_{e f f} u
$$

where $\nu_{e f f}=\nu_{i}-\nu_{a}$ is the effective ionization frequency taking into account ionization $\left(\nu_{i}\right)$ and attachment $\left(\nu_{a}\right)$ frequency. It was shown in [2] that the more exact conservative form of the diffusion term in equation (3) leads to very similar numerical results. The values for $\nu_{i}$ and $\nu_{a}$ are functions of local effective field $E_{\text {eff }}$ and are obtained in air from BOLSIG+ 8]. We can also find the expression of $\nu_{e f f}$ in the report [9]. The local effective field can be written as :

$$
E_{e f f}=\frac{E_{r m s}}{\sqrt{1+\omega^{2} / \nu_{c}^{2}}}
$$

where $E_{r m s}$ is the local root mean square of the electrical field, and $\omega$ is the angular frequency of the incident field.

$D_{\text {eff }}$ is an effective diffusion coefficient defined as

$$
D_{e f f} \simeq \frac{\alpha D_{e}+D_{a}}{1+\alpha}, \quad \alpha=\nu_{i} \frac{\varepsilon_{0}}{q u \mu_{e}}
$$

where $\nu_{i}$ is the ionization frequency, $D_{e}=\mu_{e} k_{B} T_{e} / q$ is the free electron diffusion coefficient and $D_{a}=\mu_{i} k_{B} T_{e} / q$ is the ambipolar diffusion coefficient. $T_{e}$ is the electron temperature (in Kelvin), $\mu_{e}=q /\left(m_{e} \nu_{c}\right)$ is the electron mobility, $\mu_{i}$ is the ion mobility and $k_{B}$ is the Boltzmann constant. It was shown heuristically in [1] and confirmed numerically in [7] that the effective diffusion coefficient above can satisfactorily describe the fact that the plasma front, where the electron and ion densities are small and the Debye length is large, diffuses with the free 
electron diffusion coefficient while the plasma bulk diffusion is ambipolar. We assume in the simulations that $k_{B} T_{e}$ is constant and equal to $2 \mathrm{eV}$, and that $\mu_{i}=10^{-2} \mu_{e}\left(\right.$ so that $\left.D_{a}=10^{-2} D_{e}\right)$ [2].

For more details about this physical model, we can see the $\mathrm{PhD}$ thesis of Guo Quang Zhu [10] and the PHD thesis of K. Kourtzanidis [11.

\section{FDTD approximation}

To solve Maxwell's equations in time domain, a finite-difference method (FDTD) was developed in 1966 by K. S. Yee [12. It is currently one of the most famous method used to solve these equations. To solve our coupled Maxwellplasma problem, we are going to adapt the Yee scheme to our system of equations formed by (1), (2), (3) :

$$
\left\{\begin{array}{l}
\epsilon_{0} \frac{\partial \mathbf{E}}{\partial t}-\nabla \times \mathbf{H}=-\mathbf{J} \\
\mu_{0} \frac{\partial \mathbf{H}}{\partial t}+\nabla \times \mathbf{E}=0 \\
\mathbf{J}=q u \mathbf{v} \\
\frac{\partial u}{\partial t}-\operatorname{div}\left(D_{e f f} \nabla u\right)=\nu_{e f f} u \\
\frac{\partial \mathbf{v}}{\partial t}+\nu_{c} \mathbf{v}=\frac{q}{m_{e}} \mathbf{E} \\
u=0 \quad(x, y) \in \partial \Omega
\end{array}\right.
$$

The Yee's scheme is based on a leap-frog scheme in both space and time. We define the computation domain $\Omega$ as a parallelepiped where some absorbing boundary conditions are applied to emulate the infinite space [13.

In our case, we consider an uniform cartesian mesh and we split $\Omega$ such that :

$$
\Omega=[0, L x] \times[0, L y] \times[0, L z]=\bigcup_{i, j, k} \Omega_{i, j, k}
$$

where $\Omega_{i, j, k}=\left[x_{i}, x_{i+1}\right] \times\left[y_{j}, y_{j+1}\right] \times\left[z_{k}, z_{k+1}\right]$ so that $x_{i}=(i-1) \Delta x, y_{j}=$ $(j-1) \Delta y, z_{k}=(k-1) \Delta z$, for $1 \leq i \leq N_{x}, 1 \leq j \leq N_{y}, 1 \leq k \leq N_{z}$, where $\Delta x=L x / N_{x}, \Delta y=L y / N_{y}, \Delta z=L z / N_{z}$ and $N_{x}, N_{y}, N_{z} \in \mathbb{N}^{*}$. 
The discretization of the Maxwell equations by the Yee scheme is well known and has been developed in many papers. For a complete study of this scheme, we can see, for example, the book of Taflove [14. Here and for an easier understanding, we recall that the Yee scheme is used to simulate the following system of equations :

$$
\left\{\begin{array}{c}
\varepsilon \frac{\partial \mathbf{E}}{\partial t}-\nabla \times \mathbf{H}=-\mathbf{J} \\
\mu_{0} \frac{\partial \mathbf{H}}{\partial t}+\nabla \times \mathbf{E}=0
\end{array}\right.
$$

where the unknowns $\left(E_{x}, E_{y}, E_{z}\right)$ and $\left(H_{x}, H_{y}, H_{z}\right)$ of the scheme are located as shown in Figure (1) and evaluated at time $t=n \Delta t$ for the electric fields and $t=(n+1 / 2) \Delta t$ for the magnetic fields.

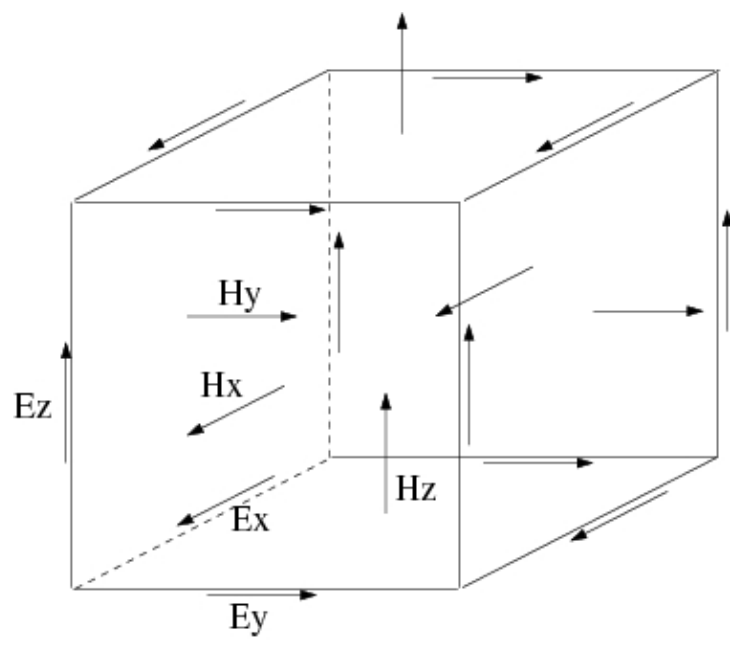

Figure 1: Localisation of the unknown in then FDTD approximation.

More precisely, the components evaluated by the scheme are given by :

$$
\mathbf{E}_{i, j, k}^{n}=\left(\begin{array}{c}
E_{x_{i+\frac{1}{2}, j, k}}^{n} \\
E_{y_{i, j+\frac{1}{2}, k}}^{n} \\
E_{z_{i, j, k+\frac{1}{2}}}^{n}
\end{array}\right), \mathbf{H}_{i, j, k}^{n+1 / 2}=\left(\begin{array}{c}
H_{x_{i, j+\frac{1}{2}, k+\frac{1}{2}}^{n+1 / 2}}^{n+1 / 2} \\
H_{y_{i+\frac{1}{2}, j, k+\frac{1}{2}}}^{n+1 / 2} \\
H_{z_{i+\frac{1}{2}, j+\frac{1}{2}, k}}^{n+1 / 2}
\end{array}\right)
$$

where, for a given component $U, U_{i, j, k}^{n}$ defines the value of this component taken at the time $t=n \Delta t$ and at the location $x=i \Delta x, y=j \Delta y, z=k \Delta z$, where 
$\Delta t, \Delta x, \Delta y, \Delta z$ define respectively the steps in time and space domains. Finally, the Yee scheme is explicit and of two order in time and in space when homogeneous step sizes are considered. The well-known drawbacks of this scheme are the staircase representation of the objects and its significant numerical dispersive error. In free space, the scheme is stable under the condition [14] :

$$
\Delta t \leq \frac{1}{\nu} \frac{1}{\sqrt{\frac{1}{\Delta x^{2}}+\frac{1}{\Delta y^{2}}+\frac{1}{\Delta z^{2}}}}
$$

where $\nu=1 / \sqrt{\varepsilon_{0} \mu_{0}}$ defines the velocity of the waves inside the medium.

\subsection{Approximation of the plasma equations}

Consider the plasma's equations given in the physical model by :

$$
\left\{\begin{array}{l}
\frac{\partial u}{\partial t}-\nabla \cdot\left(D_{e f f} \nabla u\right)=\nu_{e f f} u \\
\frac{\partial \mathbf{v}}{\partial t}+\nu_{c} \mathbf{v}=\frac{q}{m_{e}} \mathbf{E}
\end{array}\right.
$$

In our finite differences approximation, the unknowns are the plasma density $u$ and the velocity $\mathbf{v}=\left(v_{x}, v_{y}, v_{z}\right)$. By considering the same mesh as for the electromagnetic fields, we choose to locate the plasma density at the mesh nodes while the velocity position is identical to the one of electric field in the Yee scheme (see Figure 2).

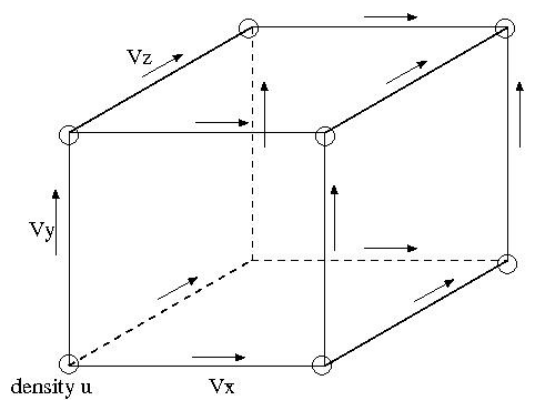

Figure 2: Unknown position related to the plasma in the cell grid

By using the same notation for the plasma unknowns as for the electromagnetic fields, the global finite differences discretization for the velocity equation 
is given by :

$$
\frac{\mathbf{v}_{i, j, k}^{n+1 / 2}-\mathbf{v}_{i, j, k}^{n-1 / 2}}{\Delta t}+\nu_{c} \frac{\mathbf{v}_{i, j, k}^{n+1 / 2}+\mathbf{v}_{i, j, k}^{n-1 / 2}}{2}=\frac{q}{m_{e}} \mathbf{E}_{i, j, k}^{n}
$$

with

$$
\mathbf{v}_{i, j, k}^{n+1 / 2}=\left(\begin{array}{c}
v_{x_{i+\frac{1}{2}, j, k}}^{n+1 / 2} \\
v_{y_{i, j+\frac{1}{2}, k}}^{n+1 / 2} \\
v_{z_{i, j, k+\frac{1}{2}}}^{n+1 / 2}
\end{array}\right)
$$

and for the diffusion equation which describes the plasma density, we have :

$$
\frac{u_{i, j, k}^{n+1}-u_{i, j, k}^{n}}{\Delta t}-\left(\nabla \cdot\left(D_{e f f} \cdot \nabla u\right)\right)_{i, j, k}^{n}=\nu_{e f f_{i, j, k}} u_{i, j, k}^{n}
$$

We reformulate the operator $\left(\nabla \cdot\left(D_{e f f} \nabla u\right)\right)$ as the sum of two operators:

$$
\nabla \cdot\left(D_{e f f} \nabla u\right)=\nabla D_{e f f} \cdot \nabla u+D_{e f f} \Delta u
$$

The first term on the right side of the previous equation is discretized as follows :

$$
\begin{aligned}
\left(\nabla D_{e} \cdot \nabla u\right)_{i, j, k}^{n}= & \frac{D_{e f f_{i+1, j, k}}^{n}-D_{e f f_{i, j, k}}^{n}}{x_{i+1}-x_{i}} \frac{u_{i+1, j, k}^{n}-u_{i, j, k}^{n}}{x_{i+1}-x_{i}} \\
+ & \frac{D_{e f f_{i, j+1, k}}^{n}-D_{e f f_{i, j, k}}^{n}}{y_{j+1}-y_{j}} \frac{u_{i, j+1, k}^{n}-u_{i, j, k}^{n}}{y_{j+1}-y_{j}} \\
& +\frac{D_{e f f_{i, j, k+1}}^{n}-D_{e f f_{i, j, k}}^{n}}{z_{k+1}-z_{k}} \frac{u_{i, j, k+1}^{n}-u_{i, j, k}^{n}}{z_{k+1}-z_{k}}
\end{aligned}
$$

and the discretization of the second term is given by :

$$
\begin{aligned}
\left(D_{e f f} \Delta u\right)_{i, j, k}^{n}=D_{e f f_{i, j, k}}^{n} & {\left[\frac{2\left(u_{i+1, j, k}^{n}-u_{i, j, k}^{n}\right)}{\left(x_{i+1}-x_{i}\right)\left(x_{i+1}-x_{i-1}\right)}+\frac{2\left(u_{i-1, j, k}^{n}-u_{i, j, k}^{n}\right)}{\left(x_{i}-x_{i-1}\right)\left(x_{i+1}-x_{i-1}\right)}\right.} \\
& +\frac{2\left(u_{i, j+1, k}^{n}-u_{i, j, k}^{n}\right)}{\left(y_{j+1}-y_{j}\right)\left(y_{j+1}-y_{j-1}\right)}+\frac{2\left(u_{i, j-1, k}^{n}-u_{i, j, k}^{n}\right)}{\left(y_{j}-y_{j-1}\right)\left(y_{j+1}-y_{j-1}\right)} \\
& \left.+\frac{2\left(u_{i, j, k+1}^{n}-u_{i, j, k}^{n}\right)}{\left(z_{k+1}-z_{k}\right)\left(z_{k+1}-z_{k-1}\right)}+\frac{2\left(u_{i, j, k-1}^{n}-u_{i, j, k}^{n}\right)}{\left(z_{k}-z_{k-1}\right)\left(z_{k+1}-z_{k-1}\right)}\right]
\end{aligned}
$$

For the effective diffusion coefficient $D_{\text {eff }}$, we take :

$$
D_{e f f_{i, j, k}}^{n}=\frac{D_{a}+\alpha_{i, j, k}^{n} D_{e}}{1+\alpha_{i, j, k}^{n}} \quad \text { with } \quad \alpha_{i, j, k}^{n}=\nu_{i} \varepsilon_{0} /\left(q u_{i, j, k}^{n} \mu_{e}\right)
$$


In the case where $D_{\text {eff }}$ is constant and $\nu_{\text {eff }}=0$, the scheme $10 p$ is stable under the condition (see Appendix A) :

$$
\Delta t \leq \frac{1}{2 D_{e}} \frac{1}{\frac{1}{\Delta x^{2}}+\frac{1}{\Delta y^{2}}+\frac{1}{\Delta z^{2}}}
$$

with $\left(D_{a} \leq D_{\text {eff }} \leq D_{e}\right)$.

\subsection{Time discretization of the global problem and stability :}

In the numerical solutions of equations (5) two different time steps $\Delta t_{M}$ and $\Delta t_{u}$ are used respectively for the discretization of the Maxwell and plasma equations. This is due to the fact that the time evolution of the plasma density is very slow compared to that of the electromagnetic field which is related to the incident wave frequency.

In the equations (5), we choose to take an explicit Euler scheme, a leapfrog scheme and an implicit centred scheme to discretize in time respectively the plasma density equation, the Maxwell equations and the velocity equation. In our time approximations, we consider the second members in both Maxwell and plasma equations (5) taken at the previous time. By considering this assumption, the stability studies are independently done on every equation of the system (5) and we find two conditions on the time steps :

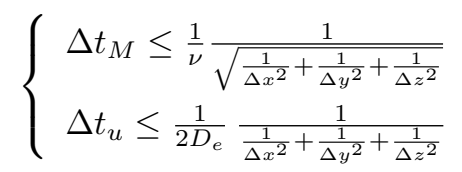

By considering our numerical experiments, theses conditions seem sufficient to ensure the stability of the numerical scheme. The global theoretical condition for the stability of our coupled systems of equations is difficult and remains an open mathematical problem which is not treated here. In our simulations, the values of $D_{e}, \nu$ and of the minimum cell dimension are such that the ratio between $\Delta t_{u}$ and $\Delta t_{M}$ is quite large. Indeed, by considering an homogeneous mesh where $\Delta_{x}=\Delta_{y}=\Delta_{z}=d l$, we have $\frac{\Delta t_{M}}{\Delta t_{u}}=\frac{D_{e}}{2 \nu d l}$. In this formula, the velocity of the waves in the medium is equal to $\nu=3 . e 8 \mathrm{~ms}$, the coefficient $D_{e}$, after calculation by considering the values previously given for $k_{B} T_{e} / q, n u_{c}$ and $n u_{e}=q /\left(m_{e} \nu_{e}\right)$, 
is lower than 1 and the step taken into account in our simulations $d l=1 e-4 m$. With these values, the ratio $\frac{\Delta t_{M}}{\Delta t_{u}}$ is of the order of 3000 . Then to reduce the computation time in our scheme, we use two time steps as in [7] [15] [6]. $\Delta t_{M}$ to compute the electromagnetic fields and $\Delta t_{u}=m \Delta t_{M}$ to evaluate plasma density, where $m$ is an integer value given by $m=\operatorname{int}\left(\frac{\Delta t_{u}^{\prime}}{\Delta t_{M}}\right) \gg 1$, where $\Delta t_{u}^{\prime}$ is obtained by the stability condition for the density plasma. In the calculation process of the electromagnetic fields, between the two steps $\Delta t_{M}$ and $m \Delta t_{M}$, the plasma density is assumed constant and equal to the value evaluated at $\Delta t_{M}$.

Concerning the velocity equation, the time step taken into account is the same as for the electromagnetic fields.

Finally the numerical scheme for the system (5), is given by :

$$
\left\{\begin{array}{l}
\mathbf{E}_{i, j, k}^{n+1}=\mathbf{E}_{i, j, k}^{n}+\frac{\Delta t_{M}}{\varepsilon_{0}}\left(\nabla_{h} \times \mathbf{H}^{n+1 / 2}-\mathbf{J}_{i, j, k}^{n+1 / 2}\right) \\
\mathbf{H}_{i, j, k}^{n+1 / 2}=\mathbf{H}_{i, j, k}^{n-1 / 2}-\frac{\Delta t_{M}}{\mu_{0}} \nabla_{e} \times \mathbf{E}^{n} \\
\mathbf{v}_{i, j, k}^{n+1 / 2}=\left[\left(1-\frac{\Delta t_{M} \nu_{c}}{2}\right) \mathbf{v}_{i, j, k}^{n-1 / 2}+\Delta t_{M} \frac{q}{m_{e}} \mathbf{E}_{i, j, k}^{n}\right] /\left(1+\frac{\Delta t_{M} \nu_{c}}{2}\right) \\
u_{i, j, k}^{N+1}=u_{i, j, k}^{N}+\Delta t_{u}\left[\left(\nabla \cdot\left(D_{e f f} \cdot \nabla u\right)\right)_{i, j, k}^{N}+\nu_{e f f_{i, j, k}} u_{i, j, k}^{N}\right] \\
\mathbf{J}_{i, j, k}^{n+1 / 2}=q \frac{u_{i, j, k}^{N}+u_{i, j, k}^{N}}{2} \mathbf{v}_{i, j, k}^{n+1 / 2}
\end{array}\right.
$$

with $t^{n}=n \Delta t_{M}$ and $t^{N}=N \Delta t_{u}=N m \Delta t_{M}$,

\section{Optimization of the simulation time}

The use of two different time steps to evaluate the electromagnetic fields and the plasma density allows to reduce the computation time, however it remains important. This is the reason why we propose a new idea to reduce the number of evaluations of the electromagnetic field by considering the monochromatic property of the source and the relatively slow plasma density evolution.

- we have two different time steps $\Delta t_{M}$ and $\Delta t_{u}$ for the discretization of the Maxwell and plasma equations,

- the plasma density is constant between $t^{N}$ and $t^{N+1}$ and equal to $u^{N}$, 
- the plasma density depends only of the $E_{r m s}$ value and is then not instantaneously dependent of the electromagnetic field $(\mathbf{E}, \mathbf{H})$,

- the electric and magnetic fields are periodic in time due to the monochromatic source.

Considering an incident monochromatic field with a period $T$ and assuming that on the computational domain $\Omega$ the electromagnetic fields have a stable permanent regime after $p$ periods (we call $p$ the parameter of optimisation).

Let $t_{N}$ be a given time where we have computed a plasma density $u^{N}$ and an electromagnetic field $\left(E^{N}, H^{N}\right)$. We want to compute these quantities at the time $t_{N+1}=t_{N}+\Delta t_{u}$, where $\Delta t_{u}$ is the time step to evaluate the plasma density. In order to optimize the computation time, the idea is to perform the following steps (see Fig. 3).

- First, we re-evaluate $\Delta t_{u}$ so that $\Delta t_{u}=k p T$, where $k$ is defined by the floor function value of $\Delta t_{u} /(p T)$;

- Evaluate the electromagnetic fields on the interval $\left[t_{N}, t_{N}+p T[\right.$, with $t_{N}+p T \ll t_{N+1}$, on the different time points given by the time step $\Delta t_{M}$, related to the Maxwell equations. In this step we consider the plasma density constant and equal to $u^{N}$;

- Evaluate the RMS field $E_{r m s}(x, y, z)=\frac{1}{T} \sqrt{\int_{t_{N}+(p-1) T}^{t_{N}+p T} E(t, x, y, z)^{2} d t}$;

- Evaluate the plasma density at the time step $t_{N+1}$;

- Evaluate the electromagnetic fields at $t_{N+1}$. By using the periodicity, the electromagnetic fields values at $t_{N+1}$ and at $t_{N}+p T$ are the same. Then, there are no calculation to do for evaluating the electromagnetic fields at $t_{N+1}$.

remark :

At each time step $N$, the electromagnetic fields are only evaluated, in the process, on the interval $\left[t_{N}, t_{N}+p T\left[\right.\right.$ and not on the interval $\left[t_{N}+p T, t_{N+1}[\right.$. This 


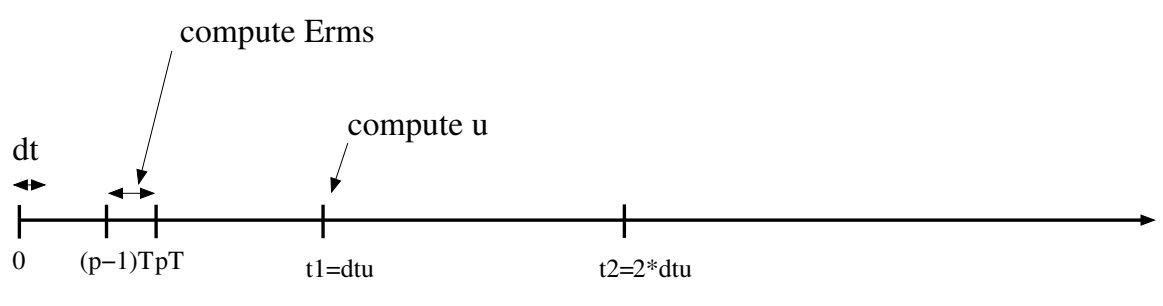

$\longleftrightarrow$

compute E,B,v

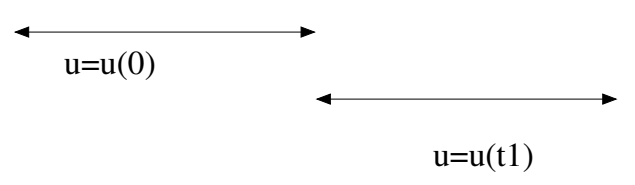

dt : time step for Maxwell

$\mathrm{dtu}$ : time step for plasma density

Figure 3: Optimized algorithm.

implies an important reduction of the computation time, if $\Delta t_{u}$ is very larger than $p T$. This last condition is generally true in our applications.

\section{Numerical results}

In this section, we present some results on the gain, in terms of computation time, obtained by our optimized algorithm for simulations on the Maxwell problem and the Maxwell-plasma problem. Concerning the Maxwell-plasma problem, we propose to study the evolution of a plasma density of a discharge at high pressure in air. To make this simulation, we consider a plane wave which illuminates an initial ball of low density plasma, in order to localize the plasma discharge in space without any focusing. The initial density is low enough that it remains transparent to the wave energy but able to later generate the breakdown.

\subsection{Description of the configuration}

145

The configuration studied consists in the scattering of a plane wave on a plasma discharge in a 3D infinite domain. We evaluate for this configuration, 
the spatial evolution of the plasma density and the electromagnetic fields along the time.

First, to study the performance of our optimized method, we consider a rectangular computational domain defined by $[0,6 \mathrm{~mm}] \times[0,3 \mathrm{~mm}] \times[0,3 \mathrm{~mm}]$ and bounded by Perfectly Matched Layers (PML) to simulate the infinite space. A monochromatic $\left(f_{0}=100 G H z\right)$ incident plane wave source is defined by $\left(k_{x}=1, E_{y}, H_{z}\right)$ where at a point $(x, y, z)$ and at the time $t$ the electric field is given by $E_{y}(x, y, z)=E_{0} \sin \left(\omega\left(t-k_{x} \cdot x / c_{0}\right)\right)$, with $E_{0}=6 M V / m, c_{0}$ is the speed of the wave in the vacuum and $\omega=2 \pi f_{0}$. The initial plasma density is localized inside a ball of center $P_{0}=(4.5 \mathrm{~mm}, 1.5 \mathrm{~mm}, 1.5 \mathrm{~mm})$ and with a radius $r=0.1 \mathrm{~mm}$. The initial value of the plasma density is equal to $u_{0}=1 . e 18 \mathrm{~m}^{-3}$. The problem is considered at atmospheric pressure $(\mathrm{pr}=760 \mathrm{Torr})$ and we want to follow the evolution of the electromagnetic fields and the plasma density at the test-point $P_{1}=(4.5 \mathrm{~mm}, 1.5 \mathrm{~mm}, 1.5 \mathrm{~mm})$. In our first simulations, we have fixed the optimisation parameter $p$ introduced in the previous section to 10 .

Next, we propose to evaluate with our approach, 3D spatial plasma density evolution on an example proposed in [16 and compare the computation time for the two methods.

\subsection{Analysis of performance: Maxwell simulations}

Considering the previous configuration, first, we show the advantage of using our optimized algorithm to evaluate only the electromagnetic fields by assuming no plasma density in the problem. In our example, we take a time step $\Delta t_{M}=2.5 e-14 s$. Figure (4) shows the fields computed by using our optimized algorithm and the non optimized method. We can see that the fields computed with both methods are in very good agreement. The great interest of our optimized algorithm is the reduction of $90 \%$ of the computation time to have a similar solution than the non optimized method.

\subsection{Analysis of performance : Maxwell-plasma simulation}

Now, in this section, we are interested in the Maxwell-plasma problem. In our simulations, we take the same time step $\Delta t_{M}$ as previously to solve the 
$\mathrm{t}$ in $[0: 10 \mathrm{~T}], \mathrm{T}=1 \mathrm{e}-11$
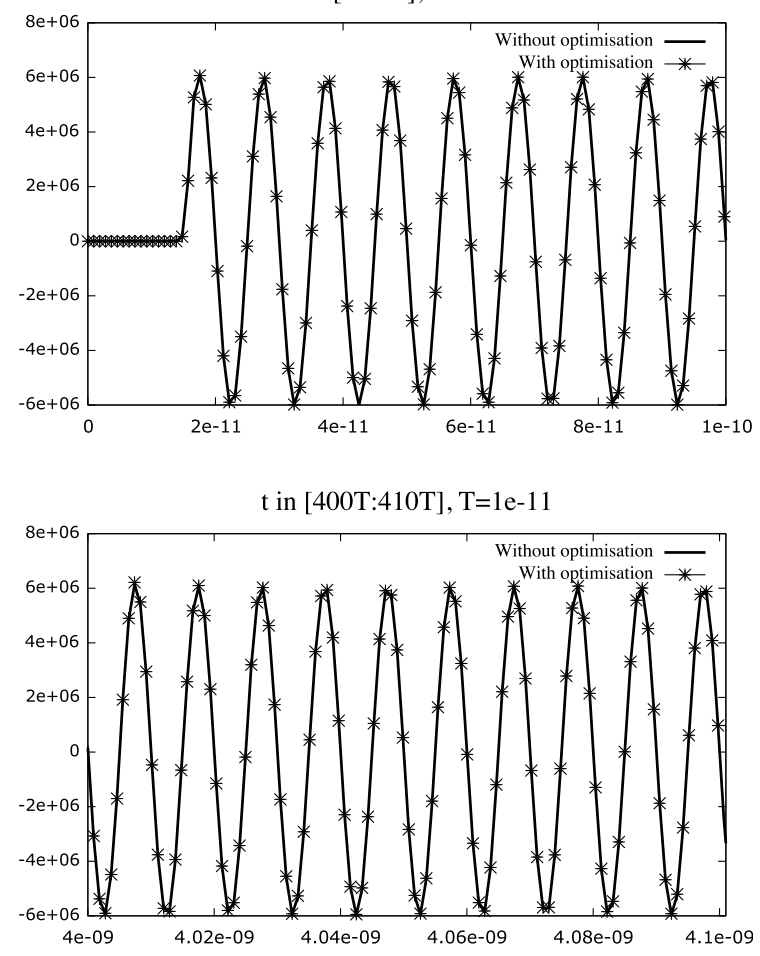

$\mathrm{t}$ in $[200 \mathrm{~T}: 210 \mathrm{~T}], \mathrm{T}=1 \mathrm{e}-1$
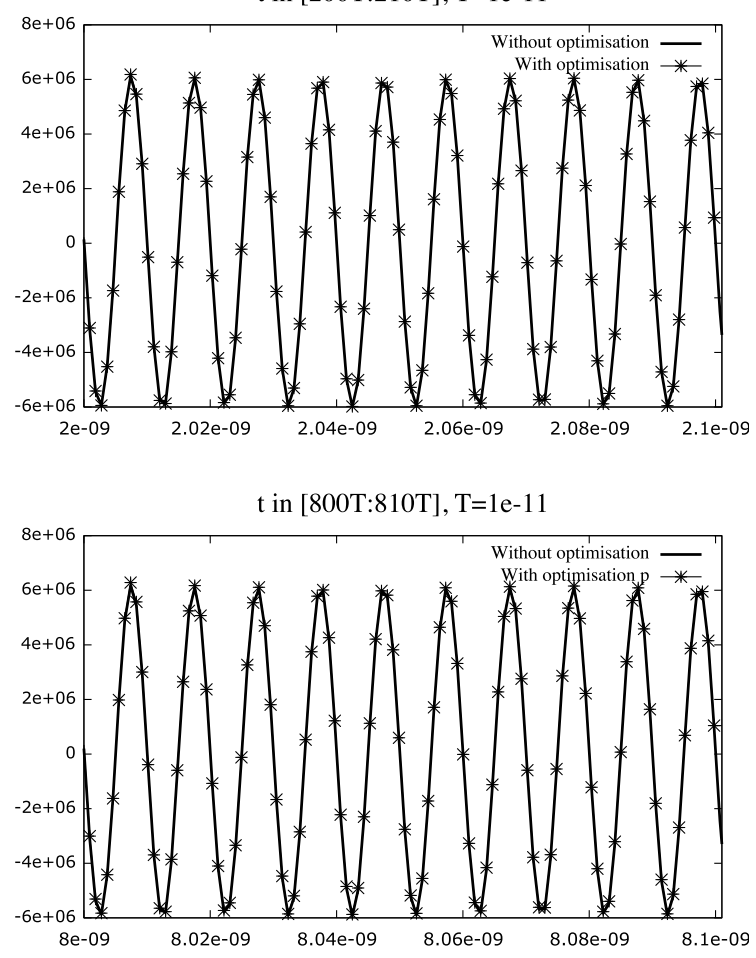

Figure 4: Evolution of the $\mathbf{E}_{y}$ component of the electric field (top: left) at the point $P_{1}$ with and without optimized algorithm. Other figures are the zoom at different times.

Maxwell problem and the following parameters to solve the plasma equations :

- a time step for the plasma $\Delta t_{u}=1 . e-9 s=100 T$, where $T$ definies the period of the incident plane wave signal ;

- $\nu_{m}=3.8 e 12 s^{-1}$.

- an initial plasma density $\left(m^{-3}\right)$

$$
\left.u_{0}(x, y, z)=1 . e 18 \exp \left(\frac{-\left(\left(x-x_{0}\right)^{2}+\left(y-y_{0}\right)^{2}+\left(z-z_{0}\right)^{2}\right)}{2 \sigma^{2}}\right)\right),
$$

with $\sigma=1 . e-5 m$ and $P_{0}=\left(x_{0}, y_{0}, z_{0}\right)=(4.5 \mathrm{~mm}, 1.5 \mathrm{~mm}, 1.5 \mathrm{~mm})$.

The Figure (5) shows the evolution of the electric field at the point $P_{1}$ obtained with and without the optimized algorithm. We can again observe a good agree- 
ment between both results. Concerning the plasma density, Figure (6) shows

the value obtained at the test-point $P_{1}$ for both approaches and the differences between these solutions obtained with and without our optimized algorithm. We note on this figure, the very good agreement between both solutions with a same gain in terms of computation time regarding the Maxwell simulation, for our optimized algorithm.

5.4. Improving the parameter of optimization $p$ in the evaluation of the electromagnetic fields

In the previous example, using the optimized algorithm, we evaluated the electromagnetic fields during a time interval equal to ten periods $(p=10)$ of the incident plane wave. In this subsection, we show that it is possible to decrease the computation time by reducing this time interval to three periods $(p=3)$, without modifying the accuracy of the solution. By taking into account the previous example where we carried out a Maxwell-plasma simulation, we make different computations by choosing a parameter of optimization $p$ equal to 3,4 and 5. This parameter is used to calculate the time interval in which we solve Maxwell's equations and the velocity's equations. Figure 7 and table 1 present the plasma density at the test-point $P_{1}$ and the computation time consumed for different values of $p$. By considering these results we notice that we can improve the computation time given in the previous example by a factor 3 . In our configuration, it was not possible to take a time interval less than 3 periods to evaluate the electromagnetic fields because they are not in a stable state. This parameter appears to be physically dependent on the non resonant behaviour of the presented configuration and the travelling time of the incident wave through the domain. It is then clearly related to each physical problem under investigation. The validation cases proposed here are therefore very favorable regarding the benefit of the optimized process. Indeed, for the study of discharges and plasma formation at atmospheric pressure due to High Power Microwave, in our applications the field amplitude and the source frequency considered are respectively greater than $3 M V$ and $1 G H z$. With these assumptions, before having a 
$\mathrm{t}$ in $[0: 10 \mathrm{~T}], \mathrm{T}=1 \mathrm{e}-11$
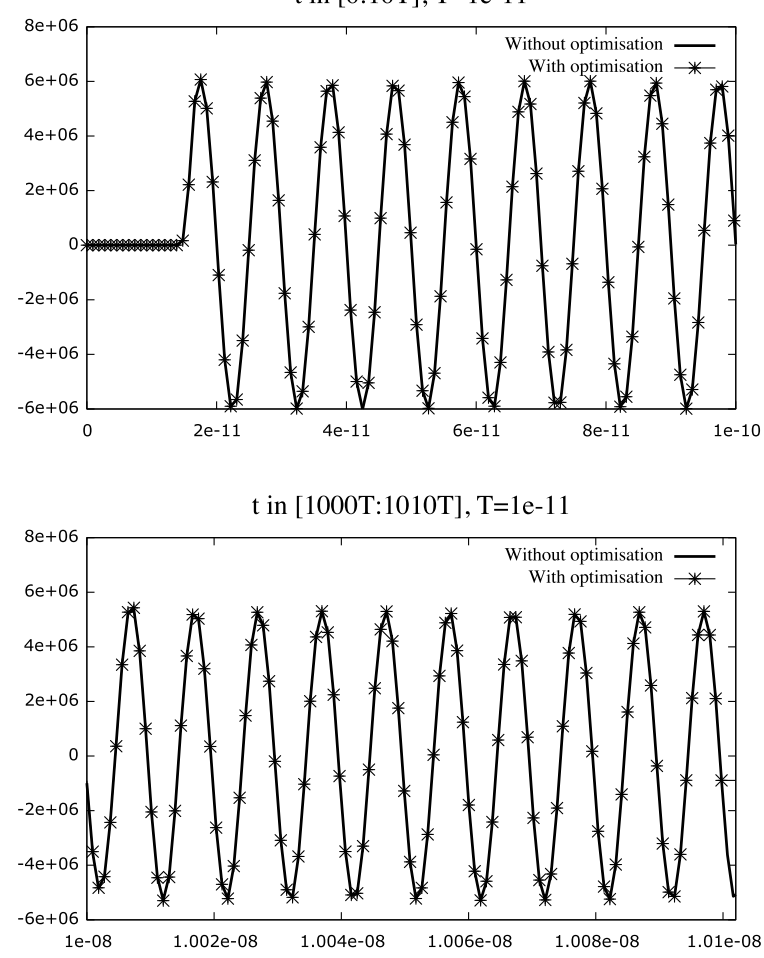

$\mathrm{t}$ in [3000T:3010T], T=1e-11

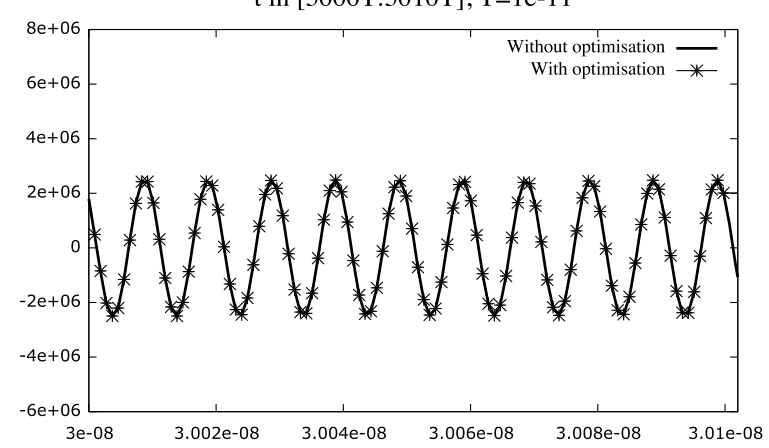

$\mathrm{t}$ in [100T:110T], T=1e-11
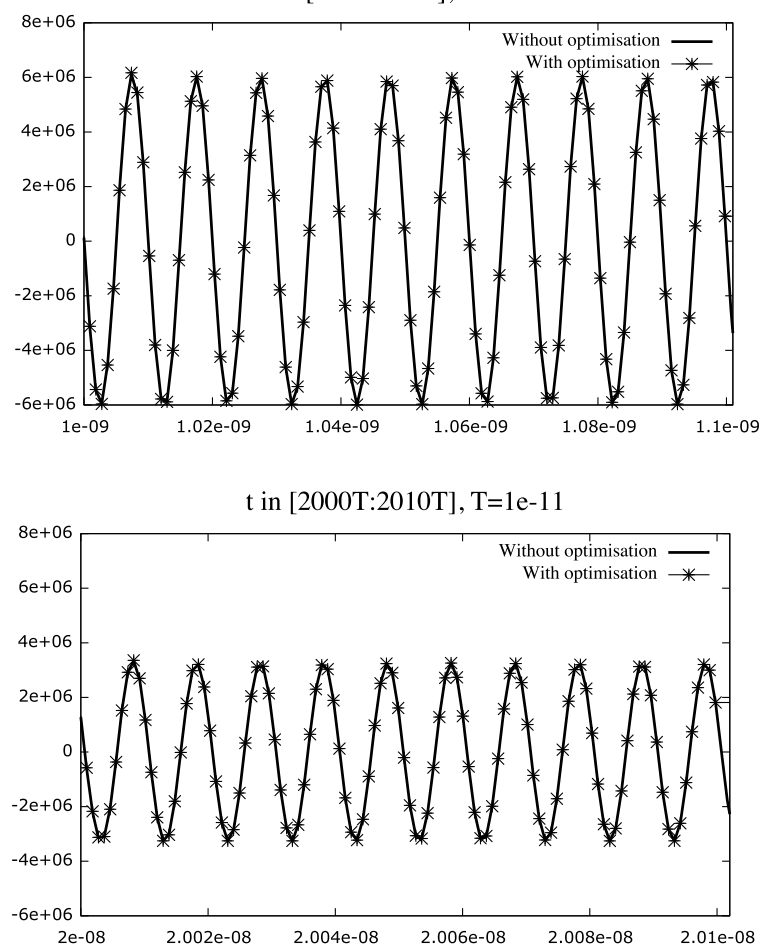

$\mathrm{t}$ in [4000T:4010T], $\mathrm{T}=1 \mathrm{e}-11$

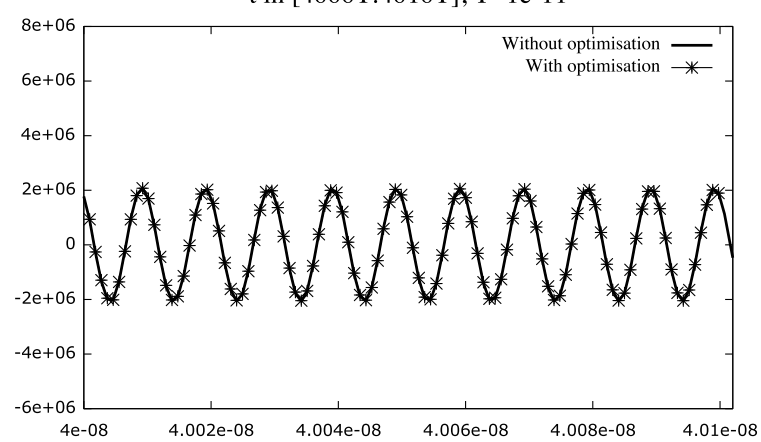

Figure 5: Evolution of the $\mathbf{E}_{y}$ component of the electric field at the test-point $P_{1}$ with and without optimized algorithm for different ranges of time.

modification of the density plasma by diffusion, the electromagnetic field travels 215 a significant number $p$ of periods (see [17]). 

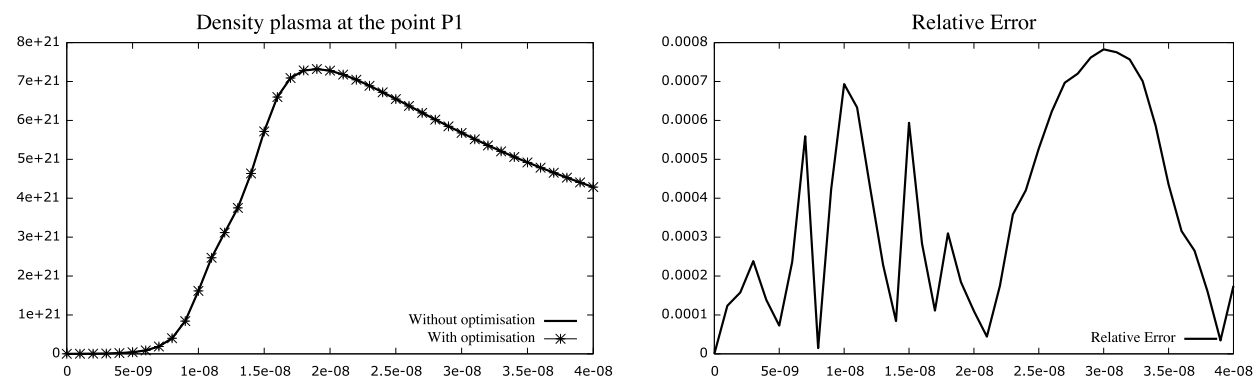

Figure 6: Evolution of the plasma density (left) and the relative error (right) at the test-point $P_{1}$ with and without optimized algorithm.

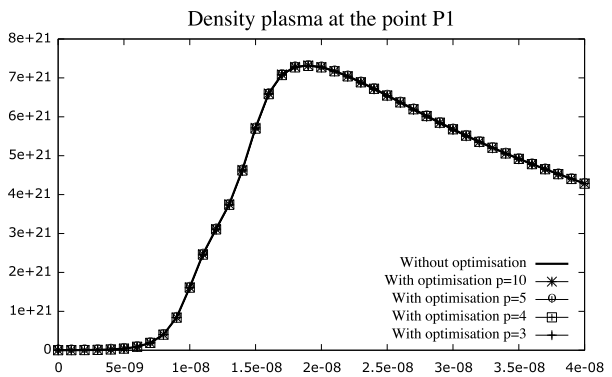

Figure 7: Evolution of the plasma density at the test-point $P_{1}$ with and without optimized algorithm by changing the parameter $p$.

\begin{tabular}{|l|c|c|c|c|r|}
\hline$p$ & 3 & 4 & 5 & 10 & 100 (without optimisation) \\
\hline CPU-time (s) & 964 & 1047 & 1448 & 2729 & 26582 \\
\hline
\end{tabular}

Table 1: Computation time as a function of $p$

\subsection{Evolution of the spatial density plasma in a discharge in air at high pressure}

To validate and to quantify the performances of our approach on a physical problem describing the plasma formation at atmospheric pressure, we propose to compared it with some results obtained in [16] with an implicit scheme to solve Maxwell-plasma equations. To have similar simulations in terms of memory and computational costs, we modify our previous configuration, by taking a source frequency equal to $f_{0}=110 \mathrm{GHz}$, and a domain of interest defined by $2 \lambda \times 2.2 \lambda \times 1.5 \lambda$, where $\lambda$ is the wavelength of the source. With this new 
configuration, we make simulations by using 64 processors and an approximation of the computational domain given by $400 \times 440 \times 300$ cells, as for [16]. Figure (8) shows the evolution of the density plasma at different time-step, obtained with our approach. We note on this figure, the same behaviour of the density plasma as in the results given in the paper [16, with moreover, an important gain in computation time. Indeed, the time-consuming with our optimized explicit method approach is approximatively equal to ten hours, against one week for the implicit scheme.
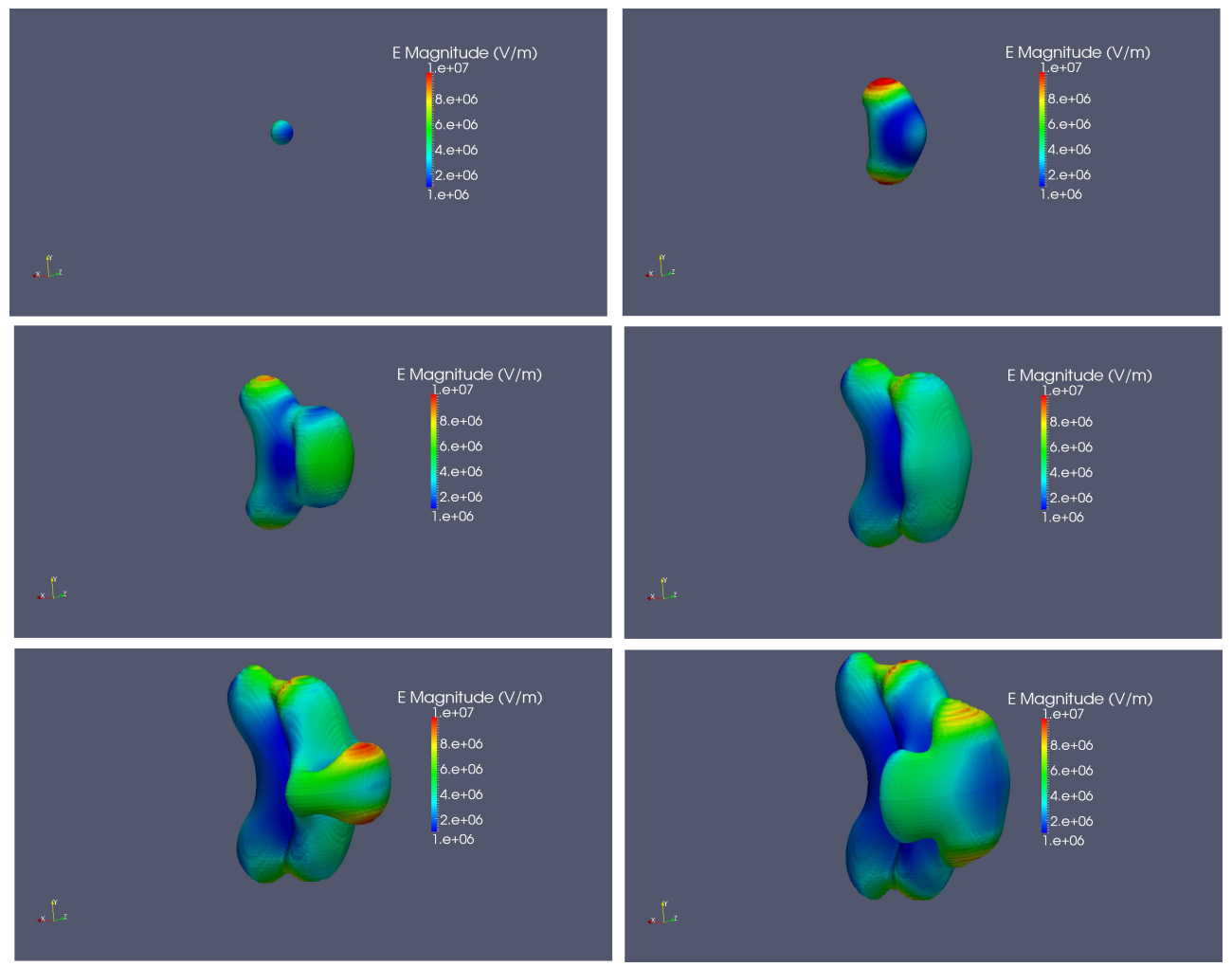

Figure 8: 3D-spatial evolution of the electron density isosurface $\left(1 . e 21 \mathrm{~m}^{-3}\right)$ contours coloured by the electric fields, at $19 n s, 27 n s, 38 n s, 50 n s, 59 n s$ and $70 n s$. 


\section{Conclusion}

In this paper, we proposed and validated an optimized algorithm to efficiently compute electromagnetic fields and plasma densities in a coupled MaxwellPlasma problem where the source is a monochromatic HPM that ignites the breakdown. This new algorithm permits to obtain for 3D simulations an important gain in terms of computation time. This gain is higher when dealing with limited size domain, in terms of wavelengths, and non, or low, resonant cases. Because using the conventional FDTD method without this optimization is difficult and sometimes impossible to properly handle a 3D Maxwell-plasma problem, the proposed process is of great interest. With our optimized algorithm, it is now possible to hope handling larger problems and investigating specific 3D non linear evolutions of plasmas and electromagnetic fields.

\section{Acknowledgement}

The first author thanks Taibah university for her research funding. This is an extension of a previous work funded by PLASMAX project.

\section{References}

[1] J. P. Boeuf, B. Chaudhury, G. Q. Zhu, Theory and modelling of selforganization and propagation of filamentary plasma arrays in microwave breakdown at atmospheric pressure, Phys. Rev. Lett. 104 (2010) 015002.

[2] B. Chaudhury, J. P. Boeuf, G. Q. Zhu, Pattern formation and propagation during microwave breakdown, Phys. Plasma 17 (2010) 123505.

[3] K. Kourtzanidis, F. Rogier, J. Boeuf, Adi-fdtd modeling of microwave plasma discharges in air towards fully three-dimensional simulations, Computer Physics Communications 195 (2015) 49-60.

[4] J. Berenger, An implicit fdtd scheme for the propagation of vlf-lf radio waves in the earth-ionosphere waveguide, Comptes Rendus Physiques 15 (2014) 393-402. 
[5] Y. Yu, J. Niu, J. Simpson, A 3 3-d global earth-ionosphere fdtd model including an anisotropic magnetized plasma ionosphere, IEEE Trans. Antennas and Prop. 60 (7) (2012) 3246-3256.

[6] J. S. A. Samini, An efficient 3-d fdtd model of electromagnetic wave propagation in magnetized plasma, IEEE Antennas and Prop. 63 (1) (2015) $269-279$.

[7] B. Chaudhury, J. P. Boeuf, Computational study of filamentary pattern formation in a high power microwave breakdown generated air plasma, IEEE Trans, Plasma Sci. 38 (2010) 2281.

[8] G. J. M. Hagelaar, L. C. Pitchford, Solving the boltzmann equation to obtain electron transport coefficients and rate coefficients for fluid models, Plasma sources science and technology 4 (2005) 722.

[9] S. D. N. Larry K Warme, Roy E. Jorgenson, Ionization coefficient approach to modeling breakdown in nonuniform geometries, Tech. Rep. SAND20034078, Sandia National Laboratory, Albuquerque, New Mexico 87185 and Livermore, California 94550 (2003).

[10] G. Q. Zhu, Modeling of plasma dynamics and patterne forulation during high pressure microwave breakdown, Ph.D. thesis, University of Toulouse (France) (2012).

[11] K. Koutzanidis, Numerical simulation of plasma acuators for flow control, Ph.D. thesis, University of Toulouse (France) (2014).

[12] K. S. Yee, Numerical solution of initial boundary values problems involving maxwells equations in isotropic media, IEEE, Transport on antennas and propagation 3 (1966) 302.

[13] J. P. Berenger, A perfectly matched layer for the absorption of electromagnetic waves, J. Comput. Phys. 114 (1994) 185-200. 


\section{Appendix A}

By considering $D_{\text {eff }}=D_{e}$ constant and $\nu_{e f f}=0$, the equation 10 is given by :

$\frac{u_{i, j, k}^{n+1}-u_{i, j, k}^{n}}{\Delta t}=D_{e}\left(\frac{u_{i+1, j, k}^{n}+u_{i-1, j, k}^{n}-2 u_{i, j, k}^{n}}{\Delta_{x}^{2}}+\frac{u_{i, j+1, k}^{n}+u_{i, j-1, k}^{n}-2 u_{i, j, k}^{n}}{\Delta_{y}^{2}}+\frac{u_{i, j, k+1}^{n}+u_{i, j, k-1}^{n}-2 u_{i, j, k}^{n}}{\Delta_{z}^{2}}\right)$

with $u_{i, j, k}^{n}=U\left(t=n \Delta t, x_{i}=i \Delta x, y_{j}=j \Delta y, z_{k}=k \Delta x\right)$. To establish a condition of stability, we use the Von Neumann stability analysis by introducing in the equation (17) a modal solution given by $u_{i, j, k}^{n}=u^{n} e^{i\left(k_{x} x_{i}+k_{y} y_{j}+k_{z} z_{k}\right)}$. We obtain :

$$
\frac{U^{n+1}-U^{n}}{\Delta t}=2 D_{e} U^{n}\left(\frac{\cos \left(k_{x} \Delta x\right)-1}{\Delta x^{2}}+\frac{\cos \left(k_{y} \Delta y\right)-1}{\Delta y^{2}}+\frac{\cos \left(k_{z} \Delta z\right)-1}{\Delta z^{2}}\right)
$$

which can be written :

$$
U^{n+1}=U^{n}\left(1+2 \Delta t D_{e} A\right)
$$


with $A=\frac{\cos \left(k_{x} \Delta x\right)-1}{\Delta x^{2}}+\frac{\cos \left(k_{y} \Delta y\right)-1}{\Delta y^{2}}+\frac{\cos \left(k_{z} \Delta z\right)-1}{\Delta z^{2}}$ we can see that :

$$
1-4 \Delta t D_{e}\left(\frac{1}{\Delta x^{2}}+\frac{1}{\Delta y^{2}}+\frac{1}{\Delta z^{2}}\right) \leq 1+2 \Delta t D_{e} A \leq 1-2 \Delta t \mathbf{D}_{e}\left(\frac{1}{\Delta x^{2}}+\frac{1}{\Delta y^{2}}+\frac{1}{\Delta z^{2}}\right)
$$

by considering that $\Delta x, \Delta y, \Delta z$ are very small compared to 1 , we have :

$$
0 \leq\left|1+2 \Delta t D_{e} A\right| \leq\left|1-4 \Delta t D_{e}\left(\frac{1}{\Delta x^{2}}+\frac{1}{\Delta y^{2}}+\frac{1}{\Delta z^{2}}\right)\right|
$$

Then, in the Von Neumann analysis to ensure stability, we must have :

$$
-1+4 \Delta t D_{e}\left(\frac{1}{\Delta x^{2}}+\frac{1}{\Delta y^{2}}+\frac{1}{\Delta z^{2}}\right) \leq 1
$$

which gives

$$
\Delta t \leq \frac{1}{2 D_{e}} \frac{1}{\frac{1}{\Delta x^{2}}+\frac{1}{\Delta y^{2}}+\frac{1}{\Delta z^{2}}}
$$

